\title{
Hitting the Reset Button: An ERP Investigation of Memory for Temporal Context
}

Authors: Matthew M. Walsh ${ }^{1,2}$, Christopher A. Paynter ${ }^{1}$, Ya Zhang ${ }^{3}$ and Lynne M. Reder ${ }^{1}$

${ }^{1}$ Carnegie Mellon University, Pittsburgh, PA

${ }^{2}$ TiER1 Performance Solutions, Cincinnati, $\mathrm{OH}$

${ }^{3}$ University of Pittsburgh, Pittsburgh, PA

Corresponding Author: Matthew M. Walsh

Phone: (973) 978-4992

Email:mmw188@gmail.com

Address: Carnegie Mellon University

5000 Forbes Avenue, Baker Hall, 455G

Pittsburgh, PA 15213 


\title{
HITTING THE RESET BUTTON
}

\begin{abstract}
This study explored how temporal context influences recognition. In an ERP experiment, subjects were asked to judge whether pictures, presented one at a time, had been seen since the previous appearance of a special reset screen. The reset screen separated sequences of successively presented stimuli and signaled a change in temporal context. A "new-repeat" picture was one that had been seen before but was to be called "new" because it had not appeared since the previous reset screen. New-repeat pictures elicited a more negative FN400 component than did "old" pictures even though both had seen before during the experiment. This suggests that familiarity, as indexed by the FN400, is sensitive to temporal context. An earlier frontopolar old/new effect distinguished pictures that were seen for the first time in the experiment from all other pictures. The late positive component (LPC), which is typically greater for old stimuli, was smaller for new-repeat pictures than for pictures seen for the first time in the experiment. Finally, individual differences in task performance were predicted by the differences in amplitude of P3b that was evoked by the onset of the reset screen.
\end{abstract}

Key words: recognition, ERP, familiarity, recollection, FN400, LPC, P3b 


\section{HITTING THE RESET BUTTON}

\section{Introduction}

Studies of memory using event-related potentials (ERPs) have revealed two distinct ERP correlates of recognition. One of these is the FN400, a negative deflection at frontal sites from 300 to $500 \mathrm{msec}$, which is more negative for items judged new than for items judged old (Curran, 2000; Curran \& Cleary, 2003; Duzel, Yonelinas, Mangun, Heinze, \& Tulving, 1997; Rugg \& Curran, 2007; Rugg \& Yonelinas, 2003; Tsivilis, Otten, \& Rugg, 2001; Yovel \& Paller, 2004). The other is the late positive component (LPC), a positive deflection at parietal sites from 400 to $800 \mathrm{msec}$, which is more positive for items judged old than for items judged new (Curran, 2000; Curran \& Cleary, 2003; Duzel et al., 1997; Rugg \& Curran, 2007; Rugg \& Yonelinas, 2003; Tsivilis, Otten, \& Rugg, 2001; Yovel \& Paller, 2004). A common view in the literature is that these two components correspond to qualitatively distinct recognition memory processes (Rugg \& Curran, 2007). The FN400 corresponds to a familiarity process that provides information about whether an item has been seen, but not the context in which it appeared. In contrast, the LPC corresponds to a recollection process that does involve retrieval of contextual information (Curran, 2000; Duzel et al., 1997; Rugg \& Yonelinas, 2003).

In most dual-process models of recognition memory, familiarity is not sensitive to the context in which an item appeared (e.g. Diana, Yonelinas, \& Ranganath, 2007; Eichenbaum, Yonelinas, \& Ranganath, 2007; Jacoby, 1991; Montaldi \& Mayes, 2010; Parks \& Yonelinas, 2007; Reder et al., 2000; Yonelinas, 2002). However, there is some debate, even among dualprocess theorists, about whether contextual information might contribute to familiarity in some circumstances. Some have suggested, for example, that familiarity is sensitive to the slowly changing global context defined by the experimental setting (Mandler, 1980). In most studies of recognition memory, participants are asked to judge whether commonplace items were 


\section{HITTING THE RESET BUTTON}

encountered during the experiment. Familiarity judgments along with the FN400 differ for old and new items, though all of the items have likely been encountered many times before the experiment.

Behavioral work has shown that familiarity is also sensitive to local context (i.e., features that may vary from trial to trial). Presenting contextual features at test that have been seen repeatedly with other items during study increases spurious familiarity judgments (Diana, Peterson, \& Reder, 2004). Additionally, instructing people to encode contextual information along with items in a way that promotes unitization increases the impact of context on familiarity judgments (Diana, Yonelinas, \& Ranganath, 2008; Diana, Van den Boom, Yonelinas, \& Ranganath, 2011).

The ERP methodology has been used to provide converging evidence that local context impacts familiarity. According to one theory (Ecker, Zimmer, \& Groh-Bordin, 2007; Ecker, Zimmer, Groh-Bordin, \& Mecklinger, 2007), intrinsic (intra-item) contextual features such as object color are automatically processed and bound to object tokens, whereas extrinsic features such as background shape are not (for a review, see Zimmer \& Ecker, 2010). The absence of a match between the current stimulus and stored object tokens produces an FN400. Consequently, the FN400 is only sensitive to intrinsic local context. In an experiment that tested this theory, participants studied items presented in various colors (Ecker, Zimmer, \& Groh-Bordin; 2007). At test, items appeared in the same color or in a different color. Subjects were asked to judge whether they had studied the object, ignoring its color. The FN400 was more negative when objects appeared in a different color than when they appeared in the same color. In a second condition of the experiment, participants studied items presented on different background shapes. The manipulation of item-shape match during the test phase did not affect the FN400. Ecker et 


\section{HITTING THE RESET BUTTON}

al. interpreted these results in terms of the differential impact of intrinsic and extrinsic local context on familiarity processing.

Subsequent studies have identified other contextual features that do and do not affect familiarity. The FN400 is sensitive to changes from study to test in object color (Ecker, Zimmer, \& Groh-Bordin; 2007), word font color (Nyhus \& Curran, 2009), and object screen location (Speer \& Curran, 2007). The FN400 does not appear to be sensitive to the presence of a background shape (Ecker, Zimmer, \& Groh-Bordin; 2007), border color (Ecker, Zimmer, \& Groh-Bordin, 2007; Speer \& Curran, 2007), or superposition of an object upon a background image (Ecker, Zimmer, Groh-Bordin, \& Mecklinger, 2007). Some of these results can be understood in terms of the distinction between intrinsic and extrinsic local context. However, it is often difficult to define which features are intrinsic and extrinsic to a stimulus in advance.

\section{Is the FN400 sensitive to temporal context?}

Given that familiarity and the FN400 are influenced by at least some sources of contextual information, we asked whether the FN400 is sensitive to temporal context. We manipulated temporal context by presenting participants with sequences of images and occasionally inserting a boundary - in the form of a "reset" screen - between successive images. Participants were asked to judge whether each image had appeared since the last reset screen. Thus, the task involved location-based (i.e. temporal position relative to a boundary) rather than distance-based (i.e. elapsed time from present) judgments (Friedman, 1993).

Theories of episodic memory propose that episodes are bound with features of the context in which they appear, including temporal context (Tulving, 1983). Temporal context may change gradually over time, or with the insertion of pauses or boundaries between adjacent events. The insertion of such boundaries produces grouping effects in memory, whereby items 


\section{HITTING THE RESET BUTTON}

that appeared before or after a boundary are grouped together more strongly than items that were separated by a boundary (for a review, see Farrell, 2012). Computational models that employ a multidimensional representation of temporal distinctiveness based on distance (i.e. elapsed time from present) and location (i.e. time relative to a boundary) can account for these results (Farrell, 2012; Lewandowsky, Brown, Wright, \& Nimmo, 2006). Based on the finding that inserting a boundary between adjacent events affects subsequent episodic recollection, we asked whether manipulating temporal context would impact familiarity processing and the FN400 as well.

To address this question, we modified a delayed-match-to-sample task used with primates (Holscher \& Rolls, 2002). Holscher and Rolls recorded from perirhinal cortex in macaques as they performed the task. Neurons in the perirhinal cortex reacted to the first presentation of each picture as if it were completely new, even if the picture had appeared several times in the previous block of trials. The perirhinal cortex is thought to be involved in familiarity processing (see Diana et al., 2007 and Eichenbaum et al., 2007 for reviews), and has been identified as a potential source of the FN400 (Curran, Tepe, \& Piatt, 2006; but see Rugg \& Curran, 2007 for an alternate view). On the basis of those results, we hypothesized that the FN400 in humans would also be sensitive to when a stimulus last appeared relative to a signal that denoted the start of a new block of trials.

In our modified version of the delayed-match-to-sample task, subjects viewed a series of pictures of everyday items, along with an occasional "reset" screen. With each presentation, they judged whether the picture had appeared since the most recent reset screen. The trials of greatest interest were those where a picture that had appeared before was shown for the first time in the current block of trials (i.e. new-repeats). The key question was whether the FN400 to newrepeats would differ from the FN400 to other pictures that had already appeared in the current 


\section{HITTING THE RESET BUTTON}

block of trials (i.e. old pictures). Both types of pictures had appeared in the global context established by the experimental setting, but new-repeats had not appeared since the reset screen. Therefore, if the FN400 were more negative for new-repeat pictures than for old pictures, it would indicate that this component is sensitive to temporal context.

\section{Results}

\subsection{Behavioral analyses}

Response accuracy and correct response times are shown in Table 1. A repeated measures ANOVA revealed a significant difference in accuracy across the four picture types, $F(3,57)=$ $30.50, p<0.001$. Subjects responded more accurately to new-first pictures than to all other pictures (all $p<.001$ ). Response accuracy did not vary across the remaining picture types (all $p>$ .05). An ANOVA for correct response times also revealed a main effect of picture type $F(3,57)$ $=15.28, p<0.001$. Responses to new-first and new-repeat pictures were slower than responses to old-first and old-repeat pictures (all $p<.01$ ). Response durations did not different between new-first and new-repeat pictures, $t(19)=.43, p>.1$, nor between old-first and old-repeat pictures, $t(19)=1.98, p>.05$.

\subsection{ERP analyses}

2.2.1 Neural responses to picture stimuli. We performed a stimulus-locked analysis of ERP data from trials with correct responses. A preliminary analysis showed no main effect of block length, and no significant interactions involving block length. We collapsed data across blocks of different lengths, and generated stimulus-locked waveforms for the four types of pictures: new-first, new-repeat, old-first, and old-repeat.

176 to $260 \mathrm{msec}$. We began by examining possible perceptual priming effects evident in the early ERPs (Fig. 2). To do so, we analyzed mean ERP amplitudes from 176 to $260 \mathrm{msec}$ 


\section{HITTING THE RESET BUTTON}

(Curran \& Dien, 2003). A 4 (condition) x 3 (region: Frontal, Central, Posterior) ANOVA revealed main effects of condition, $F(3,57)=15.27, p<.001$, and region $F(2,38)=49.49, p<$ .001 . The interaction between condition and region was significant, $F(6,114)=6.07, p<.001$. Voltages were more negative for new-first pictures than for all others (Fig. 2). These effects were greatest over the frontal region (Table 2). We therefore performed an additional 2 (Old, New) x 2 (First, Repeat) ANOVA using data from frontal region. Both main effects were significant $($ Old/New: $F(1,19)=19.67, p<.001$; First/Repeat: $F(1,19)=28.54, p<.001)$, as was the interaction, $F(1,19)=7.70, p<.05$. Paired contrasts confirmed that voltages were more negative for new-first pictures than for all others (all $p<.001$ ).

300 to 500 msec. We then analyzed the ERP data during the time window of the FN400, from 300 to 500 msec (Mollison \& Curran, 2012; Speer \& Curran, 2007). A 4 (condition) x 3 (region: Frontal, Central, Posterior) ANOVA revealed main effects of condition, $F(3,57)=$ $36.58, p<.001$, and region $F(2,38)=41.89, p<.001$. The interaction between condition and region was significant, $F(6,114)=6.72, p<.001$. Voltages were most negative for new-first pictures, followed by new-repeat pictures, old-first pictures, and old-repeat pictures (Figs. 2 and 3). These effects were greatest over the frontal region (Table 3) consistent with the FN400.

We performed an additional 2 (Old, New) x 2 (First, Repeat) ANOVA using data from frontal region. Both main effects were significant $(\mathrm{Old} / \mathrm{New}: F(1,19)=52.597, p<.001$;

First/Repeat: $F(1,19)=17.68, p<.001)$, as was the interaction, $F(1,19)=8.71, p<.01$. Paired contrasts showed that voltages were more negative for new-first pictures than for all other types of pictures (all $p<.001$ ). Voltages were also more negative for new-repeat pictures than for oldfirst and old-repeat pictures (all $p<.01$ ). Voltages did not differ between old-first and old-repeat pictures, $t(19)=.89, p>.3$. 


\section{HITTING THE RESET BUTTON}

It is possible that these effects depended on the number of trials between successive repetitions of the stimulus rather than the primary manipulation of interest (i.e. whether the stimulus had appeared since the previous reset screen). To test this possibility, we replicated these analyses on the subset of trials where the stimulus had last appeared within the previous 10 trials. Voltages remained more negative for new-repeat than for old-first pictures, $t(19)=2.22, p$ $<.05$, or old-repeat pictures, $t(19)=3.36, p<.01 .^{1}$

400 to 800 msec. Next, we analyzed the ERP data during the time window of the LPC (Mollison \& Curran, 2012; Speer \& Curran, 2007), from 400 to $800 \mathrm{msec}$. A 4 (condition) x 3 (region: Frontal, Central, Posterior) ANOVA revealed a main effect of condition, $F(3,57)=$ $4.73, p<.01$, and region $F(2,38)=55.24, p<.001$. The interaction was not significant, $F(6$, $114)=1.33, p>.2$. Voltages were more positive for old pictures than for new pictures, and for first presentations than for repeat presentations (Figs. 2 and 3).

We performed an additional 2 (Old, New) x 2 (First, Repeat) ANOVA using data from posterior region (Table 4). Both main effects were significant $(\mathrm{Old} / \mathrm{New}: F(1,19)=5.61, p<$ .05 ; First/Repeat: $F(1,19)=16.59, p<.001)$, but the interaction was not, $F(1,19)=1.17, p>.2$.

\footnotetext{
${ }^{1}$ New-repeats and old-firsts provide an interesting additional contrast since the stimuli in the former case occur before and after the reset screen, whereas the stimuli in the latter case occur only after the reset screen. A 2 (condition; New-first, Old-Repeat) x 2 (number of intervening items; 10 or fewer, 10 or more) revealed a main effect of condition, $F(1,19)=9.50, p<.01$, but not of number of intervening items, $F(1,19)=.17, p>.5$. The interaction was also not significant, $F(1,19)=.12, p>.5$. New-firsts were accompanied by a uniformly more negative FN400 across both levels of lag.
} 


\section{HITTING THE RESET BUTTON}

Paired contrasts showed that LPC was less positive for new-repeats than for all other picture types (all $p<.05$ ), and that the LPC did not vary among the other picture types.

The timing of the LPC appeared to vary by condition (Figure 2). This raises the possibility that mean amplitude measured over a fixed time window could obscure differences among conditions. To address this concern, we determined when the LPC was maximal over the posterior region for each participant and in each condition. There was a significant effect of condition on peak latency, $F(3,57)=4.37, p<0.01$, with shorter latencies for old-repeat pictures than for any other type of picture (Table 5; all $p<.05)$. Peak latencies did not differ among the remaining picture types (all $p>.1$ ). To control for differences in peak latency, we calculated the peak amplitude of the LPC over the posterior region during this time window (Table 5). The effect of condition was significant, $F(3,57)=6.38, p<0.001$, owing to the smaller LPC for new-repeat pictures versus all other pictures (all $p<.05)$. The LPC was numerically, but not significantly, smaller for new-first pictures than for old-first and old-repeat pictures. Thus, the results were largely consistent with the earlier analysis of mean amplitude from 400 to $800 \mathrm{msec}$.

2.2.2 Neural responses to distractor and reset screens. We compared stimulus-locked waveforms for reset and distractor screens (Figures 4 and 5). The P3b was analyzed over the time window of 300 to $500 \mathrm{msec}$. There were significant main effects of condition, $F(1,19)=$ 7.09, $p<0.05$, and region, $F(2,38)=19.22, p<0.01$. The interaction between condition and region was also significant, $F(2,38)=3.26, p<.05$. The $\mathrm{P} 3 \mathrm{~b}$ was larger for reset screens than for distractor screens, and the difference was greatest over the posterior region (Table 6).

We examined how the amplitude of the P3b, measured over the posterior region, correlated with task accuracy. P3b amplitudes following reset and distractor screens were positively associated with overall accuracy (reset screen: $r=0.46, p<0.05$; distractor screen: $r=$ 


\section{HITTING THE RESET BUTTON}

$0.36, p<0.1$ ). Table 7 shows correlations broken out by trial type. Of the various trial types, $\mathrm{P} 3 \mathrm{~b}$ amplitude following reset and distractor screens was most predictive of response accuracy to oldfirst and old-repeat pictures.

\section{Discussion}

This study yielded several quite clear results. First and foremost, the FN400 for repeated pictures that appeared for the first time in a block of trials (new-repeat) was of intermediate value: it was more positive than for pictures that appeared for the first time in the experiment (new-first), but more negative than for pictures that were repeated within a block (old-first and old-repeat). Second, an earlier frontopolar old/new effect distinguished between pictures seen for the first time in the experiment and all other pictures. Third, the amplitude of the LPC for newrepeat pictures was more negative than for all other pictures, including those not previously seen in the experiment. Fourth and finally, the reset screen produced a greater P3b compared to the distractor screen, and P3b amplitude was positively associated with participants' performance. We discuss each of these findings in turn.

FN400. The main purpose of this experiment was to determine whether the FN400 is sensitive to temporal context. To vary temporal context, we intermittently inserted boundaries between sequences of successively presented images. Inserting such boundaries produces groupings among items in episodic memory. Computational models account for these groupings in terms of the distinct temporal contexts bound to each episode (Farrell, 2012; Lewandowsky, Brown, Wright, \& Nimmo, 2006). In addition to affecting subsequent recollection, the change in temporal context might also impact familiarity processing and the FN400. We found that the FN400 was indeed sensitive to temporal context. This is consistent with, and goes beyond earlier studies showing that the FN400 is sensitive to associative recognition, item color, font color, and 


\section{HITTING THE RESET BUTTON}

screen location (Ecker, Zimmer, \& Groh-Bordin, 2007; Mollison \& Curran, 2012; Nyhus \&

Curran, 2009; Speer \& Curran, 2007).

These results are partially consistent with a study by Holscher and Rolls (2002) where macaques performed a delayed-match-to-sample task. Neurons in the perirhinal cortex responded to the first presentation of each picture in a block as if it were new. The FN400 to new-repeats in our subjects was in the direction of responses to pictures that were entirely new. The partial reset of the FN400 could indicate that the signal arises from a heterogeneous population of neurons in the perirhinal cortex with different response properties (Xiang \& Brown, 1998). Alternatively, the FN400 could arise from other prefrontal regions that are impacted in part by earlier familiarity processing in the perirhinal cortex (Rugg \& Curran, 2007). Both accounts permit the FN400 to be simultaneously sensitive to temporal context and other sources of contextual information. We interpret the intermediate FN400 for new-repeats in our experiment in terms of their match to the global experiment context but not to the local temporal context. Old-firsts and old-repeats matched both contexts and were most positive during the time window of the FN400, whereas new-firsts matched neither and were most negative during the time window of the FN400.

The perirhinal cortex contains a heterogeneous population of neurons with distinct response properties (Xiang \& Brown, 1998). Some are sensitive to the very first presentation of a stimulus (i.e. novelty), others are sensitive to stimuli that repeat after a brief delay (i.e. recency), and still others are sensitive to stimuli that appear more often (i.e. familiarity). The FN400 in our experiment showed something of a novelty response: it was most negative for items when they appeared for the very first time in the experiment. The FN400 was less sensitive to recency. When dividing trials by condition and number of intervening items, the effect of condition was 


\section{HITTING THE RESET BUTTON}

significant but the effect of number of intervening items and the interaction were not. Lastly, the FN400 was sensitive to familiarity, defined as whether an item had appeared since the reset screen (c.f. Holscher \& Rolls, 2002) rather than the lifetime frequency of an item (c.f. Xiang \& Brown, 1998).

Several computational models of the parahippocampal cortices - including the perirhinal cortex - have been proposed. Some hold that the perirhinal cortex performs strictly acontextual processing (Bogacz, Brown, \& Giraud-Carrier, 2001), whereas others allow varying degrees of contextual or associative processing (Meeter, Myers, \& Gluck, 2005; Norman \& O’Reilly, 2003). Our results, and the results of Holscher and Rolls (2002), are most consistent with a model by Meeter et al. (2005) in which parahippocampal nodes integrate contextual and stimulus information. Stimuli are represented along with contextual elements. The response to a stimulus is modulated by whether it has appeared before in the current context, which shifted with the reset screen in our paradigm.

Early frontopolar old/new effect. Shifting focus to the time before the FN400, we obtained a somewhat different old/new effect from 176 to $260 \mathrm{msec}$. Waveforms over the frontopolar scalp were more negative for new-first items than for all other items. Curran and Dien (2003) reported a similar effect for novel words presented in the visual modality. No such effect was obtained for words presented in the auditory modality. Tsivilis et al. (2001) also reported an early frontopolar old/new effect for images of novel scenes and objects. The effect

was obtained only when the displayed scene and image were both new, and did not distinguish between studied and rearranged pairs of scenes and images. In line with those reports, we interpret the early frontopolar effect as relating to a visually specific perceptual priming mechanism. 


\section{HITTING THE RESET BUTTON}

Late positive component (LPC). Although the primary goal of this study was to explore whether familiarity (and the FN400) is sensitive to temporal context, we also examined the LPC, the component most linked to recollection. The LPC is typically more positive when people retrieve detailed information about the stimulus, including contextual information. To correctly respond to new-repeat pictures in our task, subjects needed to know when the picture appeared relative to the reset screen. As such, we expected that the LPC would be more positive for newrepeat pictures than for new-first pictures. Contrary to this expectation, the amplitude of the LPC was more negative for new-repeat pictures than for new-first pictures.

Our interpretation of the LPC result is somewhat speculative. One explanation for the lower LPC amplitude for new-repeats versus new-firsts involves a phenomenon called the “reversed old/new” effect (Nowicka, Jednorog, Wypych, \& Marchewka, 2009). When people were instructed to try to forget words as they first studied them, LPC amplitudes during a later recognition test were lower for successfully forgotten words than for words that had not been studied (Nowicka et al., 2009). The authors attributed this "reversed old/new effect" to active inhibition of the to-be-forgotten word (see also Epstein, 1972; Geiselman, Bjork, \& Fishman, 1983; Van Hooff, Whitaker, \& Ford, 2009). Likewise, subjects in our experiment may have needed to inhibit information about having previously seen new-repeat pictures in order to correctly identify them as "new". An alternate explanation is that the lower amplitude for newrepeat pictures was driven by subjects' lower confidence. Accuracy was lowest in that condition, and response durations were longest. Previous studies show that the LPC is smaller when people are not confident in their responses (Woodruff, Hayama, \& Rugg, 2006). Additional research is needed to distinguish between these explanations of the LPC effect in our experiment. 


\section{HITTING THE RESET BUTTON}

P3b. The P3b was greater for the reset screen compared with the distractor screen. These ERP responses were particularly interesting in that they correlated positively with subjects' accuracy on the task. The most plausible explanation for this effect is that subjects differed in their attentiveness to the task, which correlated both with their P3b amplitudes and their accuracy. The P3 component is larger when people allocate more attention to a stimulus (Donchin, 1981; Kok, 2001; Polich, 2007; Wijers et al., 1989). The elevated P3b amplitudes may therefore have reflected the greater attentiveness of subjects who performed the task well. This is in line with a recent finding by Galli, Gebert, and Otten (2013) showing that subsequent memory of a word could be predicted by ERP responses to a preparatory cue, but only when attention was not divided (see also Otten et al., 2006).

A related set of findings come from the task-switching literature, where it has been found that ERP responses to a cue to switch tasks can be used to predict how quickly people will perform the new task in the upcoming block (Kieffaber \& Hetrick, 2005; Li, Wang, Zhao, \& Fogelson, 2012). In addition, a recent fMRI study (Manelis \& Reder, 2015) found that when people were shown a cue indicating the upcoming difficulty level of an n-back task, neural responses to the cue predicted their ability to perform the task accurately. In these studies, as in ours, cues did not require distinctive behavioral responses, and yet neural responses to cues were still predictive of later performance.

Conclusion. This study most clearly demonstrates the effect of temporal context on the FN400, and it indicates an effect of inhibition and/or confidence on the LPC. This study also provides support for the idea that neural processes occurring well in advance of a task can predict subsequent performance. The finding that the FN400 is sensitive to temporal context is 


\section{HITTING THE RESET BUTTON}

consistent with, and goes beyond existing behavioral and neuroimaging studies of familiarity processing. In doing so, it provides new insight into the nature of recognition memory.

\section{Methods}

\subsection{Subjects}

Twenty volunteers (13 males) participated in the experiment. They ranged in age from 19 to 31 , with a mean age of 23 . They were recruited from the Carnegie Mellon University (CMU) subject pool and the Pittsburgh community. All subjects had normal or corrected to normal vision and were treated in accordance with CMU IRB guidelines. Following a debriefing at the end of the experiment, they received research experience credit or $\$ 15.00$ as compensation.

\subsection{Stimuli and Design}

The stimuli were pictures of everyday items (squirrel, hammer, etc.) acquired from the Internet and displayed against a white background. A unique stimulus list consisting of 544 pictures with 28 reset screens and 28 distractor screens was generated for each subject. The background color of the reset and distractor screens were red and blue, with color assignment randomly determined for each subject. Every picture trial could be categorized by whether the picture had appeared since the previous reset screen, and whether the picture had appeared before during the experiment. There were four types of picture trials:

1. New-first when a picture appeared for the first time in the experiment, and the correct response was new;

2. New-repeat when a picture had last appeared before the reset screen, and the correct response was new;

3. Old-first when a picture had already appeared once since the reset screen, and the correct response was old; 


\section{HITTING THE RESET BUTTON}

4. Old-repeat when a picture had appeared before and since the reset screen, and the correct response was old.

A diagram of the different conditions is shown in Figure 1.

Each subject's stimulus list consisted of four higher-order sets, each of which contained three short blocks, two medium blocks, and two long blocks. A block was defined as the period between the appearances of reset screens. We varied block length in order to make it difficult for subjects to anticipate when the next reset screen would appear. Short blocks consisted of 12 pictures, medium blocks of 20 pictures, and long blocks of 30 pictures. For each of the four higher-order trial sets, 34 picture trials appeared in each of the four conditions (new-first, newrepeat, old-first, and old-repeat). ${ }^{2}$ Each set contained a distinct collection of pictures in order to separate any effects of task familiarity and practice with the conditions of interest. Distractor screens were inserted randomly into the stimulus list to provide a control condition against which to compare ERP responses to the reset screen.

\subsection{Procedure}

Subjects sat in an electrically shielded booth. Stimuli appeared on a CRT monitor placed behind radio-frequency shielded glass and set $50 \mathrm{~cm}$ from subjects. Some trials began with the presentation of a picture. Subjects were instructed to press the F key with their left index finger if they believed the picture had appeared since the most recent reset screen, and to press the $\mathrm{J}$ key with their right index finger if the picture had not. A buzzer sounded if the subject responded

\footnotetext{
${ }^{2}$ This created a scenario where the correct response to $2 / 3$ of previously seen pictures (newrepeat, old-first, and old-repeat) was "old". Equating the frequency of responses to pictures previously seen would have the undesirable consequence of creating an overall bias toward "new" responses.
} 


\section{HITTING THE RESET BUTTON}

incorrectly. Other trials began with the presentation of a reset or distractor screen. Subjects were instructed to press the space bar to advance. The experiment was self-paced. Following all responses, a blank white screen appeared for 100 to $300 \mathrm{msec}$, after which the next trial began.

\subsection{ERP Recording}

The EEG signal was recorded from $32 \mathrm{Ag}-\mathrm{AgCl}$ sintered electrodes (10-20 system). Additional electrodes were placed on the right and left mastoids. The right mastoid served as the reference electrode, and scalp recordings were algebraically re-referenced to the average of the right and left mastoids offline. The vertical EOG was recorded from electrodes placed above and below the orbit of the left eye, and the horizontal EOG was recorded from electrodes placed at the external canthi. Electrode impedances were kept below $5 \mathrm{k} \Omega$. The EEG and EOG signals were amplified by a Neuroscan bioampliciation system (Neuroscan Inc., Sterling, VA) with a bandpass of 0.1 to $200 \mathrm{~Hz}$ and were digitized at $1000 \mathrm{~Hz}$. The continuous data were filtered offline using a $70 \mathrm{~Hz}$ low-pass filter and down-sampled to $500 \mathrm{~Hz}$.

The EEG recording was decomposed into independent components using the EEGLAB infomax algorithm (Delorme \& Makeig, 2004). Components associated with eye blinks were visually identified and projected out of the EEG recording. The data were analyzed with respect to trial onsets (i.e. the appearance of pictures, reset screens, and distractor screens). Epochs of $1050 \mathrm{msec}$ (including a $50 \mathrm{msec}$ baseline) were extracted from the continuous recording and corrected over the prestimulus interval. Epochs containing voltages above $+100 \mu V$ or below $-100 \mu V$ were excluded from further analysis. In addition to creating topographical maps over the time windows of interest, we analyzed data from three regions of interest (ROIs): a frontal region $(\mathrm{F} 3, \mathrm{FZ}, \mathrm{F} 4)$, a central region $(\mathrm{C} 3, \mathrm{CZ}, \mathrm{C} 4)$, and a posterior region (P3, PZ, P4). For all analyses, we applied the Greenhouse-Geisser correction when factors had more than two levels. 


\section{HITTING THE RESET BUTTON}

\section{Acknowledgements}

This work was supported by the National Institute of Health grants 5RO1MH052808 and T32

MH19983 to LR. It was also supported by the National Science Foundation grant DGE0549352.

We would like to thank Xiaonan Liu, Vencislav Popov, and Anna Manelis for commenting on the paper. 


\section{HITTING THE RESET BUTTON}

\section{References}

Bogacz, R., Brown, M. W., \& Giraud-Carrier, C. (2001). Model of familiarity discrimination in the perirhinal cortex. Journal of Computational Neuroscience, 10(1), 5-23.

Curran, T. (2000). Brain potentials of recollection and familiarity. Memory \& Cognition, 28, 923-938.

Curran, T., \& Cleary A. M. (2003). Using ERPs to dissociate recollection from familiarity in picture recognition. Cognitive Brain Research, 15(2), 191-205.

Curran, T., \& Dien, J. (2003). Differentiating amodal familiarity from modality-specific memory processes: An ERP study. Psychophysiology, 40(6), 979-988.

Curran, T., Tepe, K. L., \& Piatt, C. (2006). ERP explorations of dual processes in recognition memory. In H.D. Zimmer, A. Mecklinger, A., \& U. Lindenberger (Eds.), Binding in Human Memory: A Neurocognitive Approach (pp. 467-492). Oxford University Press.

Diana, R. A., Peterson, M. J., \& Reder, L. M. (2004). The role of spurious feature familiarity in recognition memory. Psychonomic Bulletin \& Review, 11(1), 150-156.

Diana, R. A., Yonelinas, A. P., \& Ranganath, C. (2007). Imaging recollection and familiarity in the medial temporal lobe: A three-component model. Trends in Cognitive Sciences, 11(9), 379-386.

Diana, R. A., Van den Boom, W., Yonelinas, A. P., \& Ranganath, C. (2011). ERP correlates of source memory: Unitized source information increases familiarity-based retrieval. Brain Research, 1367, 278-286.

Diana, R. A., Yonelinas, A. P., \& Ranganath, C. (2008). The effects of unitization on familiarity based source memory: Testing a behavioral prediction derived from neuroimaging data. Journal of Experimental Psychology: Learning, Memory, and Cognition, 34(4), 730-740. 


\section{HITTING THE RESET BUTTON}

Donchin, E. (1981). Surprise! . . Surprise? Psychophysiology, 18, 493-513.

Duzel, E., Yonelinas, A. P., Mangun, G. R., Heinze, H. J., \& Tulving, E. (1997). Event-related potential correlates of two states of conscious awareness in memory. Proceedings of the National Academy of Sciences, U.S.A., 94, 5973-5978.

Ecker, U. K., Zimmer, H. D., \& Groh-Bordin, C. (2007a). Color and context: An ERP study on intrinsic and extrinsic feature binding in episodic memory. Memory \& Cognition, 35(6), 1483-1501.

Ecker, U. K., Zimmer, H. D., Groh-Bordin, C., \& Mecklinger, A. (2007b). Context effects on familiarity are familiarity effects of context-An electrophysiological study. International Journal of Psychophysiology, 64(2), 146-156.

Eichenbaum, H., Yonelinas, A. P., \& Ranganath, C. (2007). The medial temporal lobe and recognition memory. Annual Review of Neuroscience, 30, 123-152.

Epstein, W. (1972). Mechanisms in directed forgetting. In G. H. Bower (Ed.), The psychology of learning and motivation (Vol. 6, pp. 147-191). New York, NY: Academic Press.

Farrell, S. (2012). Temporal clustering and sequencing in short-term memory and episodic memory. Psychological Review, 119, 223-271.

Friedman, W. J. (1993). Memory for the time of past events. Psychological Bulletin, 113, 44-66.

Galli, G., Gebert, D., \& Otten, J. L. (2013). Available processing resources influence encoding related brain activity before an event. Cortex, 49, 2239-2248.

Geiselman, R. E., Bjork, R. A., \& Fishman, D. L. (1983). Disrupted retrieval in directed forgetting: A link with posthypnotic amnesia. Journal of Experimental Psychology: General, 112, 58-72. 


\section{HITTING THE RESET BUTTON}

Holscher, C., \& Rolls, E. T. (2002). Perirhinal cortex neuronal activity is actively related to working memory in the macaque. Neural Plasticity, 9, 41-51.

Jacoby, L. L. (1991). A process dissociation framework: Separating automatic from intentional uses of memory. Journal of Memory and Language, 30, 513-541.

Kieffaber, P. D., \& Hetrick, W. P. (2005). Event-related potential correlates of task switching and switch costs. Psychophysiology, 42(1), 56-71.

Kok, A. (2001). On the utility of P3 amplitude as a measure of processing capacity. Psychophysiology, 38, 557-577.

Li, L., Wang, M., Zhao, Q., \& Fogelson, N. (2012). Neural mechanisms underlying the cost of task switching: An ERP study. PLoS One, 7(7): e42233. doi:10.1371/journal.pone.0042233.

Lewandowsky, S., Brown, G. D., Wright, T., \& Nimmo, L. M. (2006). Timeless memory: Evidence against temporal distinctiveness models of short-term memory for serial order. Journal of Memory and Language, 54, 20-38.

Manelis, A., \& Reder, L. M. (2015). He who is well prepared has half won the battle: An fMRI study of task preparation. Cerebral Cortex, 25(3), 726-735.

Mandler, G. (1980). Recognizing: The judgment of previous occurrence. Psychological Review, $87(3), 252-271$.

Meeter, M., Myers, C. E., \& Gluck, M. A. (2005). Integrating incremental learning and episodic memory models of the hippocampal region. Psychological Review, 112(3), 560-585.

Mollison, M. V., \& Curran, T. (2012). Familiarity in source memory. Neuropsychologia, 50, 2546-2565. 


\section{HITTING THE RESET BUTTON}

Montaldi, D., \& Mayes, A. R. (2010). The role of recollection and familiarity in the functional differentiation of the medial temporal lobes. Hippocampus, 20, 1291-1314.

Norman, K. A., \& O'Reilly, R. C. (2003). Modeling hippocampal and neocortical contributions to recognition memory: a complementary-learning-systems approach. Psychological Review, 110(4), 611-646.

Nowicka, A., Jednorog, K., Wypych, M., \& Marchewka, A. (2009). Reversed old/new effect for intentionally forgotten words: An ERP study of directed forgetting. International Journal of Psychophysiology, 71, 97-102.

Nyhus, E., \& Curran, T. (2009). Semantic and perceptual effects on recognition memory: Evidence from ERP. Brain Research, 1283(4), 102-114.

Otten, L. J., Quayle, A. H., Akram, S., Ditewig, T. A., \& Rugg, M. D. (2006). Brain activity before and event predicts later recollection. Nature Neuroscience, 9(4), 489-491.

Parks, C. M., \& Yonelinas, A. P. (2007). Moving beyond pure signal-detection models: Comment on Wixted (2007). Psychological Review, 114, 188-201.

Polich, J. (2007). Updating P300: An integrative theory of P3a and P3b. Clinical Neurophysiology, 118, 2128-2148.

Reder, L. M., Nhouyvanisvong, A., Schunn, C. D., Ayers, M. S., Angstadt, P., \& Hiraki, K. (2000). A mechanistic account of the mirror effect for word frequency: A computational model of remember-know judgments in a continuous recognition paradigm. Journal of Experimental Psychology: Learning, Memory, \& Cognition, 26, 294-320.

Rugg, M. D., \& Curran, T. (2007). Event-related potentials and recognition memory. Trends in Cognitive Sciences, 11(6), 251-257. 


\section{HITTING THE RESET BUTTON}

Rugg, M. D., \& Yonelinas, A. P. (2003). Human recognition memory: A cognitive neuroscience perspective. Trends in Cognitive Sciences, 7(7), 313-319.

Speer, N. K., \& Curran, T. (2007). ERP correlates of familiarity and recollection processes in visual associative recognition. Brain Research, 1174, 97-109.

Tsivilis, D., Otten, L. J., \& Rugg, M. D. (2001). Context effects on the neural correlates of recognition memory: An electrophysiological study. Neuron, 31(3), 497-505.

Tulving, E. (1983). Elements of episodic memory. Clarendon Press, Oxford.

Van Hooff, J. C., Whitaker, T. A., \& Ford, R. M. (2009). Directed forgetting in direct and indirect tests of memory: Seeking evidence of retrieval inhibition using electrophysiological measures. Brain and Cognition, 71, 153-164.

Wijers, A. A., Mulder, G., Okita, T., \& Mulder, L. J. (1989). Event-related potentials during memory search and selective attention to letter size and conjunctions of letter size and color. Psychophysiology, 26, 529-547.

Woodruff, C. C., Hayama, H. R., \& Rugg, M. D. (2006). Electrophysiological dissociation of the neural correlates of recollection and familiarity. Brain Research, 1100(1), 125-135.

Xiang, J. Z., Brown, M. W. (1998). Differential neuronal encoding of novelty, familiarity and recency in regions of the anterior temporal lobe. Neuropharmacology, 37, 657-676.

Yonelinas, A. P. (2002). The nature of recollection and familiarity: A review of 30 years of research. Journal of Memory \& Language, 46, 441-517.

Yovel, G., \& Paller, K. A. (2004). The neural basis of the butcher-on-the-bus phenomenon: When a face seems familiar but is not remembered. NeuroImage, 21(2), 789-800. 


\section{HITTING THE RESET BUTTON}

Zimmer, H. D., \& Ecker, U. K. (2010). Remembering perceptual features unequally bound in object and episodic tokens: Neural mechanisms and their electrophysiological correlates. Neuroscience \& Biobehavioral Reviews, 34, 1066-1079. 


\section{HITTING THE RESET BUTTON}

\section{Figure legends}

Figure 1. Simplified diagram showing distractor and reset screens, and different picture types.

Figure 2. Stimulus-locked waveforms for new-first, new-repeat, old-first, and old-repeat

pictures. Gray regions show time intervals containing the P2, the FN400, and the LPC.

Figure 3. Headplots for new-first, old-first, new-repeat, and old-repeat pictures from 176 to 260 msec (top), 300 to $500 \mathrm{msec}$ (middle), and 400 to $800 \mathrm{msec}$ (bottom).

Figure 4. Stimulus-locked waveforms for reset and distractor screens. Gray regions show time interval containing the $\mathrm{P} 3 \mathrm{~b}$.

Figure 5. Headplots for reset and distractor screens from 300 to $500 \mathrm{msec}$. 


\section{HITTING THE RESET BUTTON}

Table 1

Mean Correct RTs (in msec) and Mean Accuracy (as \%) with SEMs in Parentheses

\begin{tabular}{lcc}
\hline Trial Type & RT & Accuracy \\
\hline New-First & $1125(56)$ & $.99(.01)$ \\
New-Repeat & $1143(57)$ & $.85(.01)$ \\
Old-First & $943(25)$ & $.88(.02)$ \\
Old-Repeat & $974(37)$ & $.88(.02)$ \\
Reset & $1129(154)$ & - \\
Distractor & $1048(102)$ & - \\
\hline
\end{tabular}




\section{HITTING THE RESET BUTTON}

Table 2

Mean Amplitude of ERP Responses to Picture Stimuli from 176 to 260 msec with SEMs in Parentheses

\begin{tabular}{lccc}
\hline & Frontal & Central & Posterior \\
\hline New-First & $-6.99(1.11)$ & $-5.89(0.98)$ & $-0.14(0.64)$ \\
New-Repeat & $-5.35(1.00)$ & $-4.01(0.81)$ & $1.57(0.64)$ \\
Old-First & $-4.06(0.99)$ & $-3.37(0.87)$ & $1.60(0.65)$ \\
Old-Repeat & $-3.81(1.01)$ & $-2.32(0.88)$ & $3.24(0.70)$ \\
\hline
\end{tabular}




\section{HITTING THE RESET BUTTON}

Table 3

Mean Amplitude of ERP Responses to Picture Stimuli from 300 to 500 msec with SEMs in Parentheses

\begin{tabular}{lccc}
\hline & Frontal & Central & Posterior \\
\hline New-First & $-6.99(1.11)$ & $-5.89(0.98)$ & $-0.14(0.64)$ \\
New-Repeat & $-5.35(1.00)$ & $-4.01(0.81)$ & $1.57(0.64)$ \\
Old-First & $-4.06(0.99)$ & $-3.37(0.87)$ & $1.60(0.65)$ \\
Old-Repeat & $-3.81(1.01)$ & $-2.32(0.88)$ & $3.24(0.70)$ \\
\hline
\end{tabular}




\section{HITTING THE RESET BUTTON}

Table 4

Mean Amplitude of ERP Responses to Picture Stimuli from 400 to 800 msec with SEMs in Parentheses

\begin{tabular}{lccc}
\hline & Frontal & Central & Posterior \\
\hline New-First & $-2.66(.73)$ & $-0.97(.59)$ & $2.41(.39)$ \\
New-Repeat & $-3.06(.68)$ & $-1.58(.57)$ & $1.38(.49)$ \\
Old-First & $-2.10(.63)$ & $-0.51(.53)$ & $2.84(.47)$ \\
Old-Repeat & $-2.70(.72)$ & $-1.09(.68)$ & $2.30(.58)$ \\
\hline
\end{tabular}




\section{HITTING THE RESET BUTTON}

Table 5

Peak Latency (in msec) and Amplitude of LPC with SEMs in Parentheses

\begin{tabular}{lcc}
\hline Trial Type & Latency & Amplitude \\
\hline New-First & $589(11)$ & $5.82(.49)$ \\
New-Repeat & $569(23)$ & $4.56(.55)$ \\
Old-First & $582(14)$ & $6.32(.64)$ \\
Old-Repeat & $521(12)$ & $6.04(.73)$ \\
\hline
\end{tabular}




\section{HITTING THE RESET BUTTON}

Table 6

Mean Amplitude of ERP Responses to Distractor and Reset Screens with SEMs in Parentheses 300 to $500 \mathrm{msec}$

Frontal Central Posterior

\begin{tabular}{llll}
\hline Reset & $6.11(1.15)$ & $9.02(1.06)$ & $12.02(1.15)$ \\
Distractor & $5.17(0.74)$ & $7.18(0.92)$ & $9.70(1.21)$
\end{tabular}


HITTING THE RESET BUTTON

Table 7

Correlations between P3b Amplitude and Task Performance

\begin{tabular}{lcc}
\hline Trial Type & Reset & Distractor \\
\hline New-First & .23 & -.05 \\
New-Repeat & .17 & -.03 \\
Old-First & .49 & .49 \\
Old-Repeat & .46 & .42 \\
\hline
\end{tabular}


Figure 2
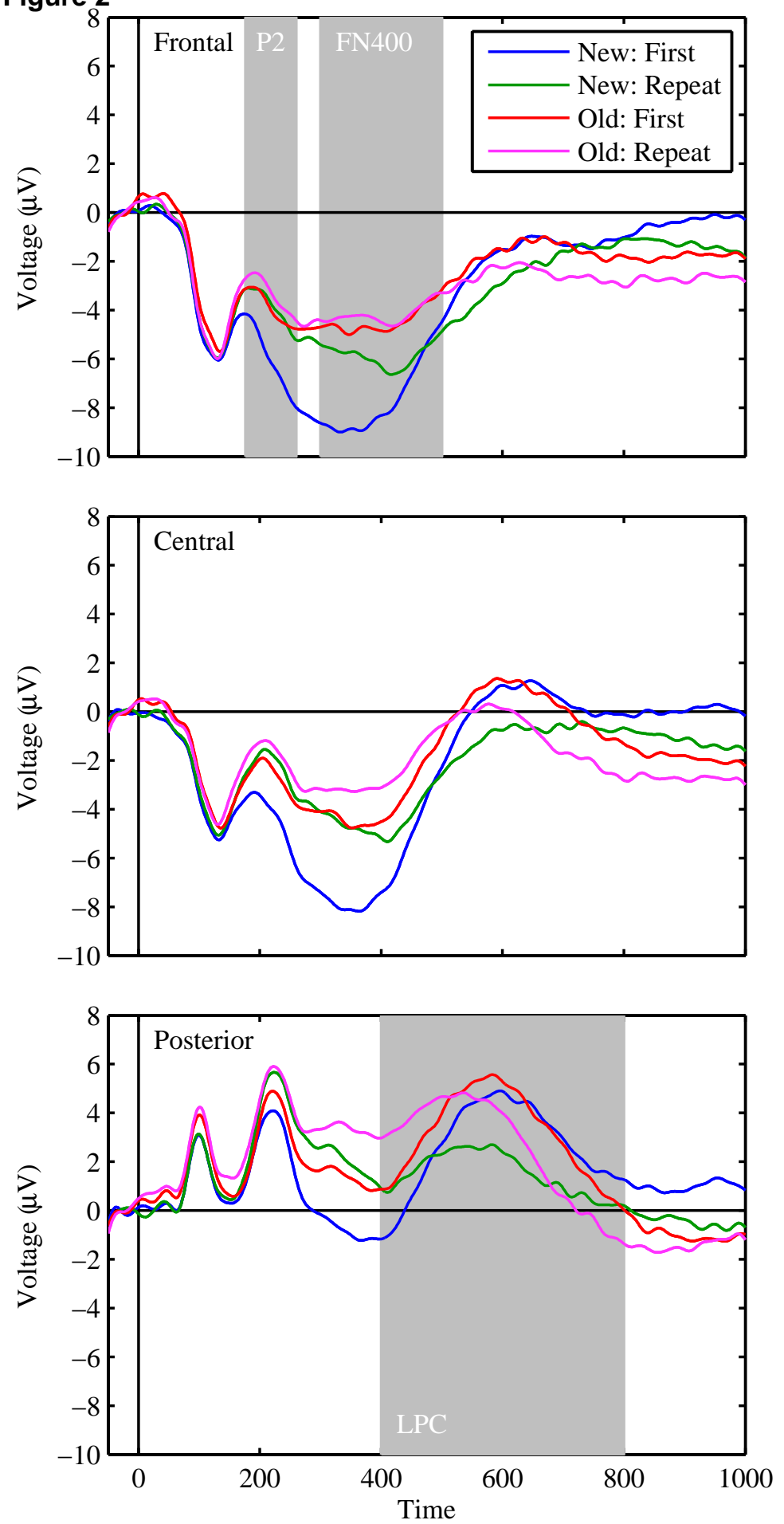
176 to $260 \mathrm{msec}$
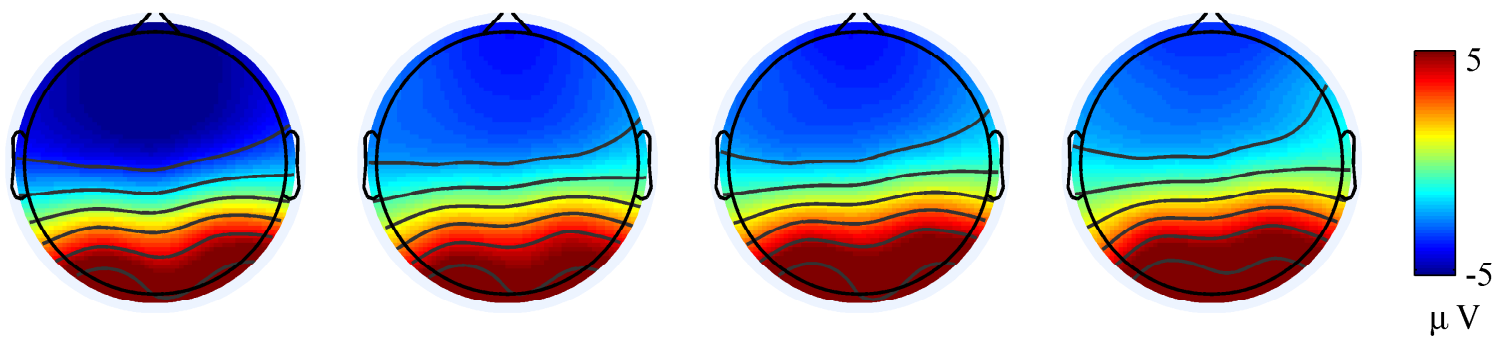

300 to $500 \mathrm{msec}$
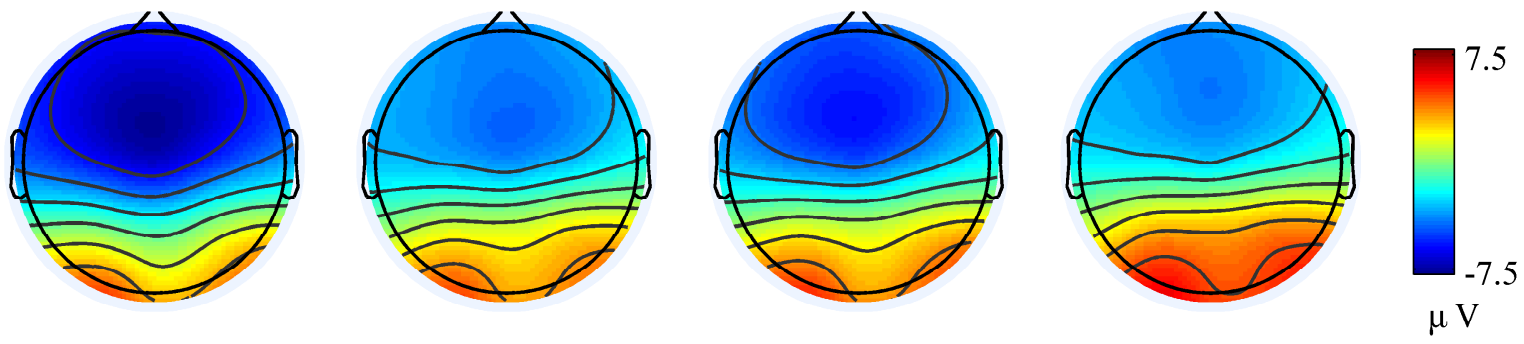

\section{0 to $800 \mathrm{msec}$}
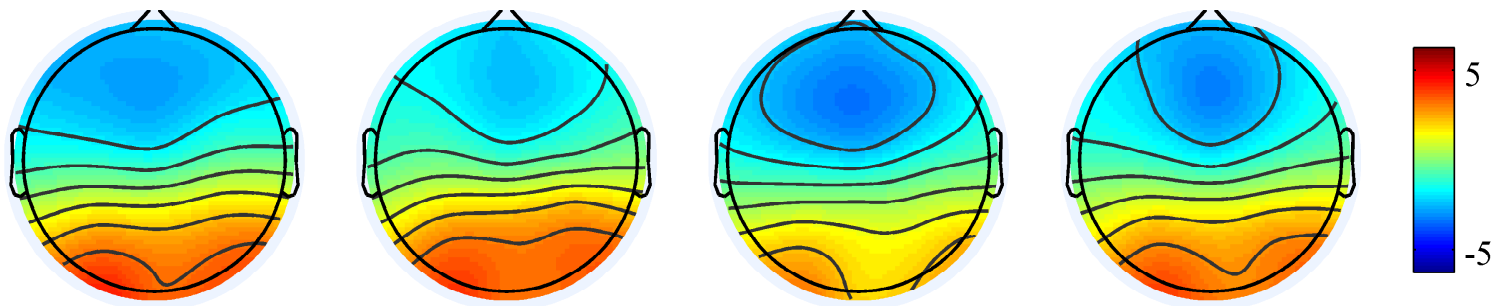

New-First

Old-First

New-Repeat

Old-Repeat 
Figure 4
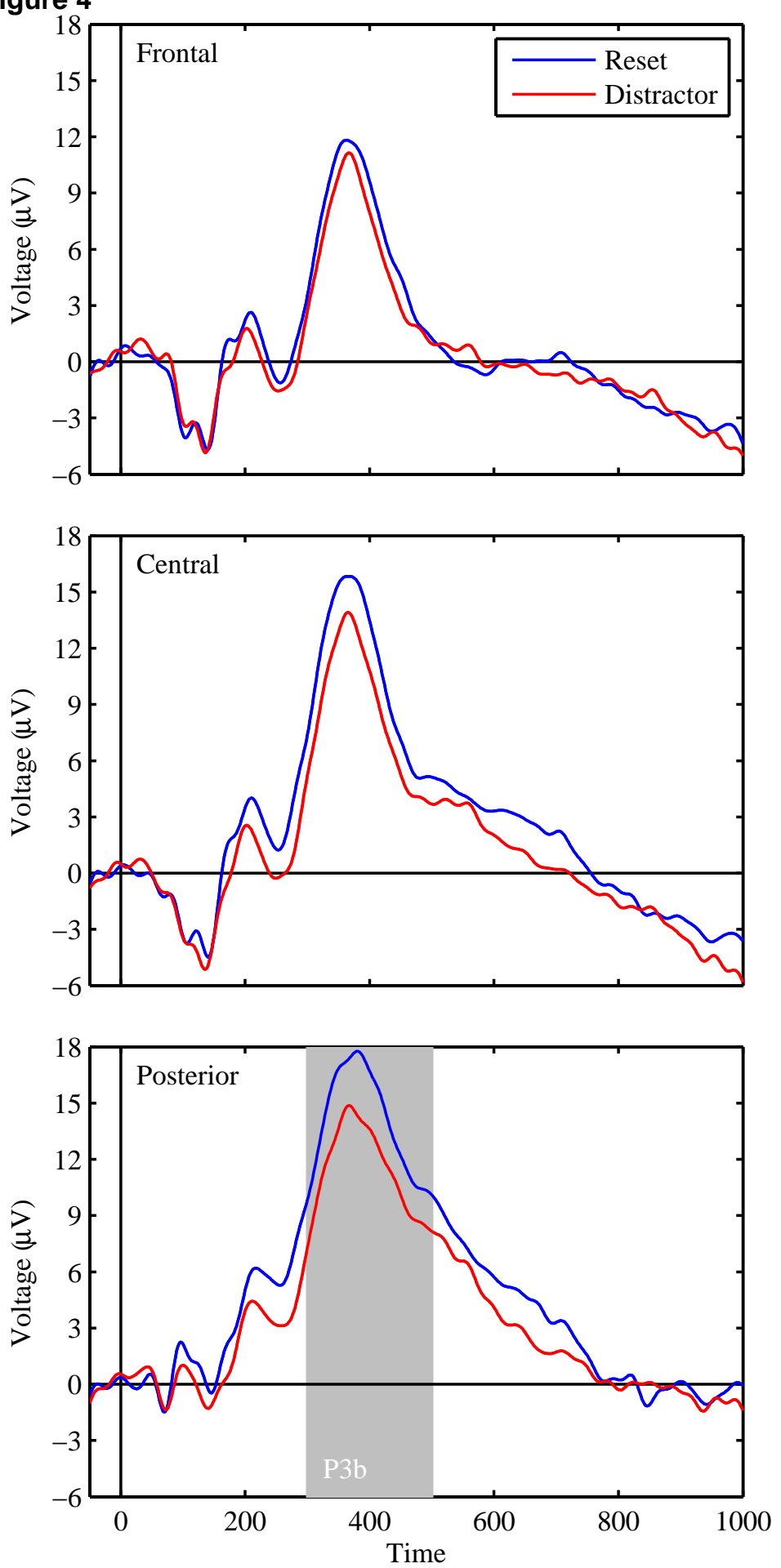
Figure 5

300 to $500 \mathrm{msec}$

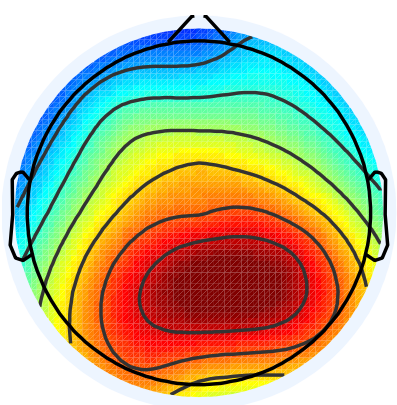

Reset

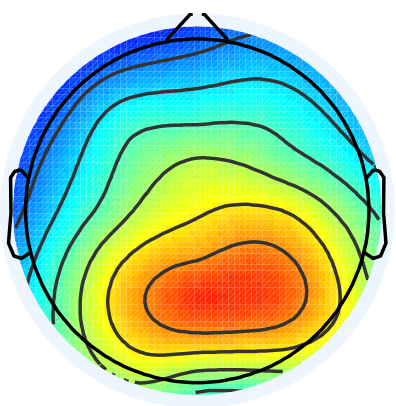

Distractor

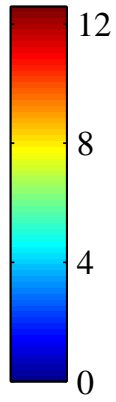

$\mu \mathrm{V}$ 